\title{
New developments in the management of advanced melanoma - role of pembrolizumab
}

REVIEW

OncoTargets and Therapy

14 September 2015

Number of times this article has been viewed

\section{Giuseppina Improta' \\ Isabella Leone' \\ Marco Donia ${ }^{2}$ \\ Stefania Gieri ${ }^{3}$ \\ Giuseppe Pelosi ${ }^{4,5}$ \\ Filippo Fraggetta ${ }^{6}$}

'Laboratory of Clinical Research and Advanced Diagnostics, IRCCS-CROB, Rionero in Vulture, Potenza, Italy;

${ }^{2}$ Center for Cancer Immune Therapy,

Department of Hematology,

Copenhagen University Hospital,

Herlev, Denmark; ${ }^{3}$ Laboratory of

Oncologic Technologies, IBFM-CNR,

Cefalù, Potenza, ${ }^{4}$ Department of

Pathology and Laboratory Medicine,

Fondazione IRCCS Istituto Nazionale

Tumori, Milan, ${ }^{5}$ Department of

Biomedical and Clinical Sciences

“Luigi Sacco", Università degli Studi

di Milano, Milan, ${ }^{6}$ Department of

Pathology, Cannizzaro Hospital,

Catania, Italy
Correspondence: Giuseppina Improta Laboratory of Clinical Research and Advanced Diagnostics, IRCCS-CROB, Via padre Pio I, 85028 Rionero in

Vulture, Potenza, Italy

Tel +39097 2726467

Email giuseppina.improta@gmail.com

\begin{abstract}
Cancer immunotherapy is now recognized to be fundamental in modern oncology, because immune system recruitment may represent a powerful and innovative strategy in cancer therapy. Pembrolizumab, a highly selective humanized monoclonal antibody directly blocking the interaction between programmed cell death-1 expressed by tumor-associated T-cells and its ligand programmed cell death-L1 present on tumor and stromal cells, was recently approved by US Food and Drug Administration for the treatment of patients with unresectable or metastatic melanoma and disease progression upon ipilimumab and BRAF inhibitor. This review will focus on the clinical development and use of pembrolizumab in the clinical practice and in the management of advanced melanoma.
\end{abstract}

Keywords: advanced melanoma, immunotherapy, PD-1 inhibitor, pembrolizumab

\section{Introduction to therapy for advanced melanoma}

Melanoma is among the less common but most deadly types of skin cancer. It arises from melanocytes and represents approximately $<5 \%$ of all cancers. It has a strong tendency to give rise to distant metastases, most commonly to lymph nodes, skin, lungs, liver, or brain. ${ }^{1}$ Over the past decade, there has been a steady increase in the incidence of melanoma worldwide, mostly related to age but disproportionately high in young adults (15-34 years); survival rates have been continually improving for the last 30 years, with an overall 5-year survival rate of $81 \%$ for men and $90 \%$ for women, likely due to earlier diagnosis. ${ }^{2}$

Across the last decades, for patients with unresectable disease, traditional chemotherapy showed no evidence of survival benefit. Until 2009, patients with American Joint Committee on Cancer stage IV melanoma had very poor prognosis, with median survival of 8-10 months. ${ }^{3}$ Developments in basic and clinical research have led to the recent introduction of new and more effective therapies in metastatic melanoma, including treatments based on the stimulation of immune response and targeted therapies. The prognosis of metastatic melanoma has recently changed due to strategies based on the immune system checkpoint inhibitor ipilimumab or several tyrosine kinase inhibitors, such as vemurafenib, dabrafenib, and trametinib. ${ }^{4-8}$ Vemurafenib and dabrafenib are selective inhibitors of BRAF V600 mutation (present in approximately $50 \%$ of melanomas), which are approved by the major regulatory bodies for the treatment of unresectable or metastatic melanoma with mutant BRAF V600. ${ }^{9,10}$ In pivotal Phase III trials, both inhibitors (independently administered) showed improved overall survival (OS), progression-free survival (PFS), and higher response rate compared with standard chemotherapy., ${ }^{4,5}$ Trametinib (an MEK inhibitor) was investigated in a randomized Phase III study as combination therapy with dabrafenib versus vemurafenib and approved by US Food and Drug Administration (FDA) in 2013 for the 
treatment of unresectable or metastatic melanoma harboring BRAF V600E or V600K mutations in combination with dabrafenib. ${ }^{11}$ Of note, trametinib also showed efficacy as monotherapy in another Phase III trial, but this compound is not currently used in this setting. ${ }^{6}$

Along with the development of BRAF and MEK inhibitors, the immunotherapy approach was improved by the introduction of ipilimumab, which is a fully human IgG1 monoclonal antibody eliciting antitumor T-cell-mediated response by interference with cytotoxic T-lymphocyte antigen-4 (CTLA-4). The drug has been approved for the treatment of metastatic melanoma, as achieved a statistically improvement in OS in two different randomized Phase III trials in pretreated and in treatment-naïve patients with metastatic melanoma, without or in combination with standard chemotherapy, respectively (even though, the latter indication is not currently used). ${ }^{7,8}$

Unfortunately, despite the introduction of these therapeutic options, the prognosis of metastatic melanoma still remains very poor. Indeed, the response rate of BRAF inhibitors exceeds $50 \%$, but median duration of response does not achieve 1 year. ${ }^{4-6,10-15}$ Most patients who respond to therapy over time develop mechanisms of acquired/secondary resistance, ultimately leading to progression of disease. In addition, approximately $15 \%$ of patients treated with BRAF inhibitors do not respond to treatment at all, likely due to intrinsic/ primary mechanisms of resistance. ${ }^{10,15}$ Conversely, immunotherapy can induce dramatic responses that are generally much more durable but unfortunately occur uncommonly (by far less than $50 \%){ }^{7,16,17}$ These data may indicate that the key to long-term tumor control can be obtained by immunotherapy, but strategies improving the likelihood of selecting patients benefiting from this therapy option need to be devised.

\section{Cancer immunotherapy}

Cancer immunotherapy is now recognized to be fundamental in modern oncology, because immune system recruitment may represent a powerful and innovative strategy in cancer therapy. ${ }^{18}$ Genetic mutations and alterations in regulatory processes of cancer cells lead to expression of various tumor-related antigens that can be presented to cytotoxic T-lymphocytes by antigen-presenting cells (APCs). In this process, pivotal is the role of T-lymphocytes in the distinction between "self" and "nonself" antigens, because immune cells have the potential to recognize cancer-related antigens as "nonself" thereby eradicating cancer cells. ${ }^{19,20}$ A major understanding of immune activation, especially T-lymphocyte activation, has identified multiple co-stimulatory and co-inhibitory pathways regulating this process. ${ }^{21}$ The two most important targets of immunotherapy are co-inhibitory receptors, such as CTLA-4 and programmed cell death-1 (PD-1) receptor, expressed on the T-lymphocyte surface. ${ }^{21}$ Both these co-inhibitory molecules serve to dampen the immune response, thus maintaining immunologic homeostasis especially during antigen presentation to T-lymphocytes resulting from a balance of stimulating and inhibiting factors to avoid uncontrolled immune activation. ${ }^{20,21}$ Unfortunately, tumor cells are capable of expressing ligands, which interact with co-inhibitory receptors to ultimately suppress tumor immunity. ${ }^{20,22}$

In the setting of clinical cancer immunotherapy, CTLA-4 and PD-1 were the first two immune checkpoints to be evaluated, differing each other in the manner and level of negative regulation of immune system. ${ }^{22}$ Specifically, CTLA-4, expressed only on T-lymphocytes, regulates negatively these cells at the initial activation level by competing with costimulatory molecule CD28 in binding B7.1 and B7.2 ligands. CTLA-4 has a greater affinity for these ligands and can also send independent inhibitory signals to T-lymphocyte. ${ }^{23-25} \mathrm{On}$ the contrary, PD-1 regulates immunity at multiple phases of the immune response, including T-lymphocyte activity in peripheral tissues (Figure 1). ${ }^{25}$

This greater understanding of immune checkpoints favored the development of several immune checkpoint inhibitors. Ipilimumab (Yervoy ${ }^{\circledR}$ ), an anti-CTLA-4 monoclonal antibody, was the first agent to demonstrate a survival benefit in patients with metastatic cutaneous melanoma and gained FDA approval in $2011 .^{7}$ However, clinical experience on the use of ipilimumab showed a response rate of only $10 \%-15 \%$ but improved OS, even though there were not negligible immune-related adverse events (diarrhea, colitis, and dermatitis) probably related to its role in the priming immune response phase. ${ }^{7,8}$ The lesson of CTLA-4 resulted in the development of other immune checkpoint inhibitors, which could be more specific, equally efficacious and immunologically less toxic. ${ }^{20}$ Consequently, the block the PD-1 signaling pathway by monoclonal antibodies able to selectively restore antitumor immunity is another appealing strategy of therapy.

Pembrolizumab (Keytruda $\left.{ }^{\circledR}\right)$, manufactured by Merck \& Co. Inc. (Whitehouse Station, New Jersey, USA), was recently approved by FDA on September 4, 2014 for the treatment of patients with unresectable or metastatic melanoma and disease progression following ipilimumab and, if BRAF V600 mutation positive, a BRAF inhibitor. This review will focus on the clinical development and use of pembrolizumab 


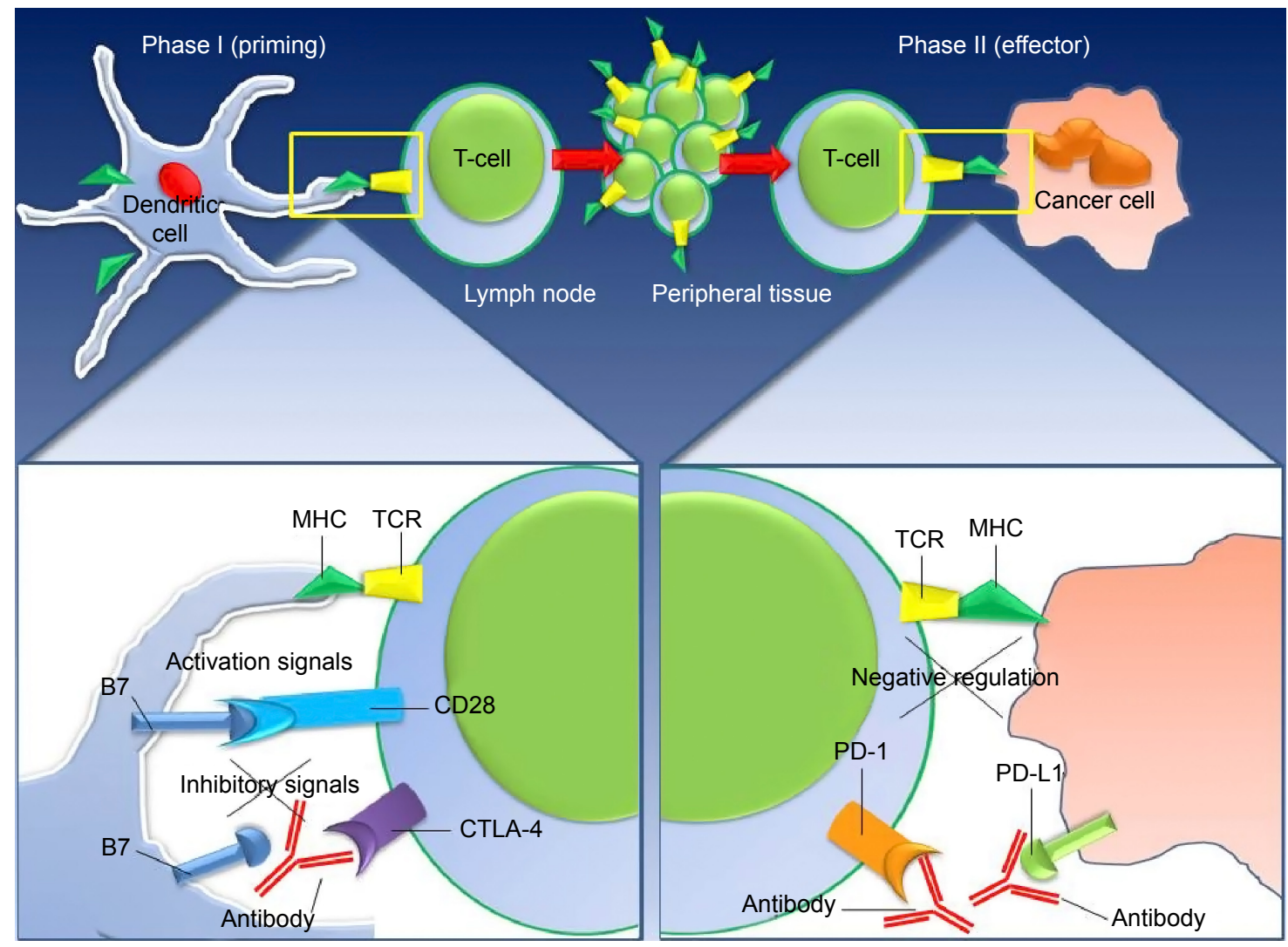

Figure I CTLA-4 and PD-I inhibitor mechanism after the activation of T-cells through their "primed" T-cell receptor, as well as a co-regulatory signal delivered by the B7 family of receptors (immune checkpoints).

Notes: CTLA-4, up-regulated shortly after activation, negatively regulates T-cell activation, binding to B7 molecules on APCs surface, during the priming phase of T-cell response within the lymph nodes. B7 molecules binding to CD28, instead, generates the opposite, activating signals. During the effector phase of T-cell immune-response, PD-I is expressed on T-cells and binds to either of its ligands (PD-LI or PD-L2), which are primarily expressed within inflamed tissues and the tumor microenvironment, resulting in inhibition of T-cell activity. Antibodies direct to CTLA-4 or PD-1/PD-LI can activate T-cells with specificity for cancer cells.

Abbreviations: MHC, major histocompatibility complex; PD-I, programmed cell death-I; CTLA-4, cytotoxic T-lymphocyte antigen-4; TCR, T-cell receptor.

in the clinical practice and in the management of advanced melanoma.

\section{Mode of action and pharmacokinetics of pembrolizumab}

Pembrolizumab is a potent and highly selective humanized monoclonal antibody of IgG4-kappa isotype, designed to directly block the interaction between PD-1 receptor, expressed on T-cells, and its ligands, PD-L1 and PD-L2, without antibody-dependent cell-mediated cytotoxicity or complement-dependent cytotoxicity. Pembrolizumab was generated by humanization of the parental murine antihuman PD-1 antibody, with an engineered Fc region for stabilization.

Specifically, the PD-1 receptor is a cell surface receptor, belonging to the CD28 family of T-cell regulators, within the immunoglobulin superfamily of receptors. ${ }^{26} \mathrm{It}$ is present on $\mathrm{CD}^{-} \mathrm{CD}^{-}$(double negative) thymocytes during thymic development and is expressed upon activation in mature hematopoietic cells, such as T-and B-cells, natural-killer cells, and monocytes, after prolonged antigen exposure. ${ }^{26}$ Through its binding to PD-L1 and PD-L2, PD-1 receptor downregulates T-cell activation and proliferation, along with downregulation of the expression of the anti-apoptotic molecule Bcl-xL, cytokine expression, and the mTOR pathway (Figure 2A). ${ }^{27,28} \mathrm{PD}-1$ receptor is highly expressed on tumor-infiltrating lymphocytes (TILs) in response to tumor antigen expression, and its binding to PD-L1 on either tumor cells or APCs, functions as an immune checkpoint to curb persistent immune response and thwarts potential injury to normal tissues. ${ }^{29}$

In particular, PD-1 is expressed on TILs of many types of cancers with its ligands (PD-L1 and PD-L2) also expressed in solid tumors (eg, melanoma, ovarian, lung, and renal carcinoma) and in different types of lymphoma, respectively..$^{30,31}$ Accumulating evidence showed, over the years, a correlation between the presence of TILs in cancer tissue and favorable prognosis in various malignancies. In particular, the presence 

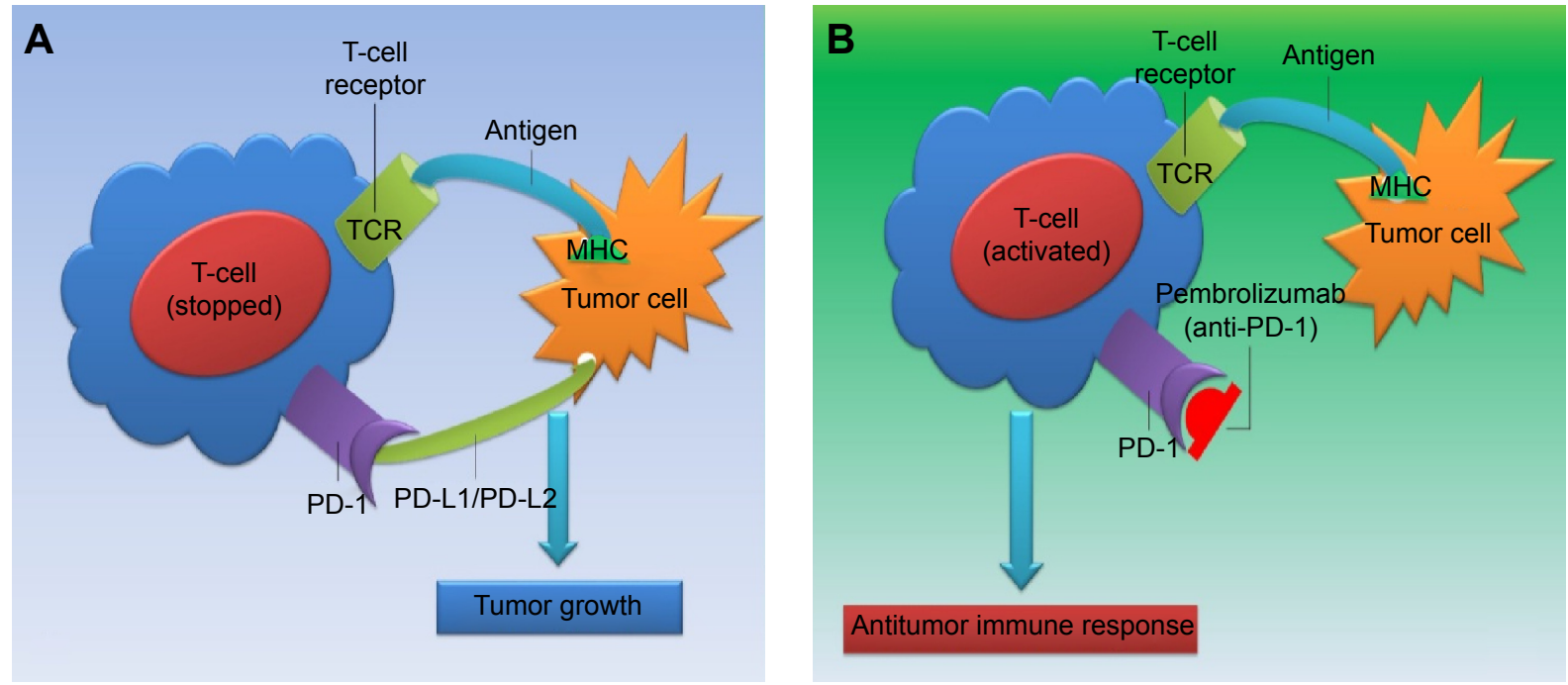

Figure 2 Tumor cell capable of resisting the immune system by expressing ligands PD-LI/PD-L2 which interact with their co-inhibitory receptor PD-I in order to suppress tumor immunity with a consequent tumor growth (A). Pembrolizumab action mechanism, according to which this anti-PD-I antibody blocks the interaction between PD-I and its ligands PD-LI and PD-L2 by binding to PD-I receptor with a consequent anti-tumor growth (B).

Abbreviations: MHC, major histocompatibility complex; PD-I, programmed cell death-I; TCR, T-cell receptor.

of $\mathrm{CD}^{+} \mathrm{T}$-cells and the ratio of $\mathrm{CD}^{+}$effector T-cells/FoxP3 ${ }^{+}$ regulatory $\mathrm{T}$-cells correlate with improved prognosis and long-term survival in solid malignancies such as ovarian, colorectal, and pancreatic cancer, hepatocellular carcinoma, melanoma, and renal cell carcinoma. ${ }^{32}$ However, expression of PD-1 and PD-L1 by TILs is associated with impaired effector function, namely, cytokine production and cytotoxic efficacy against tumor cells, and has been reported as related to poor outcome in several tumor types. ${ }^{33-38}$

Mechanisms that regulate tumor cell PD-L1 expression include the "innate immune resistance" and the "adaptive immune resistance" mechanisms being related, in the context of cancer immunology, to oncogenic signaling pathways in the microenvironment or the expression of tumor cell PD-L1 induced in response to immune activity. ${ }^{39,40}$

Using the adaptive immune resistance, tumor cells take advantage of the PD-1/PD-L1 interaction that, under normal conditions, protects cells from immune-mediated harm. Inflammatory signals, such as interferons, produced by an active antitumor immune response, in the tumor microenvironment, can lead to increased expression of PD-L1, which, in turn, protects the tumor cells. ${ }^{22,38}$ In addition, chronic antigen exposure can lead to persistent and enhanced PD-1 expression, leading to a change of the duration of T-lymphocyte-APC interaction and to T-lymphocyte anergy or exhaustion. ${ }^{22,41}$ Expression of PD-L1 has been correlated with prognosis in many types of cancer, supporting the hypothesis that PD-L1 expression may be a mechanism for tumor immune evasion. ${ }^{42-44}$ Interruption of interaction between PD-1 and its ligands is the molecular mechanism on the basis of pembrolizumab action.

Pembrolizumab is actually used for treatment of patients with unresectable or advanced melanoma, refractory to ipilimumab, as approved by FDA in September 2014. Binding to the PD-1 receptor and, consequently, blocking its interaction with ligands, PD-L1 and PD-L2, it releases PD-1 pathway-mediated inhibition of the immune response, including the antitumor immune response (Figure 2B). In practice, blocking PD-1 activity is believed to prevent inhibition of T-cell immune surveillance of tumors and, in some models, has resulted in decreased tumor growth.

Anti-PD-1 antibody pembrolizumab was tested in clinical studies in patients with advanced melanoma, and an overall median PFS, longer than 7 months, and a sustained tumor regression were reported. ${ }^{45}$ The first dose-escalation Phase I study, involving patients with solid tumors, showed the serum concentrations of pembrolizumab, in samples obtained before and after administration of the drug, lower, by a factor of approximately 5 in patients receiving $2 \mathrm{mg} / \mathrm{kg}$ every 3 weeks, than in those receiving $10 \mathrm{mg} / \mathrm{kg}$ every 3 weeks. ${ }^{46}$ Steady state through concentrations resulted $20 \%$ greater in the patients receiving $10 \mathrm{mg} / \mathrm{kg}$ every 2 weeks than in those receiving the same dose every 3 weeks; moreover, the increase in trough serum concentrations over time was consistent with the half-life of pembrolizumab of approximately 2-3 weeks. ${ }^{46}$ Pembrolizumab has been approved for intravenous injection 
at $2 \mathrm{mg} / \mathrm{kg}$ every 3 weeks, since there did not appear to be significant differences between the dosages and schedules evaluated. Elimination half-life of this agent is 26 days, and so far, no clinically important differences have been noted in its clearance in patients with renal impairment or mild hepatic impairment.

\section{Clinical trials - efficacy}

Pembrolizumab, as an anti-PD-1 monoclonal antibody, has demonstrated its useful activity in initial clinical trials for the treatment of patients with advanced melanoma evaluated in both ipilimumab-naïve and previously treated patients, contributing to its approval. ${ }^{45,47,48}$ On September 4, 2014, in fact, this promising PD-1, PD-L1/PD-L2 axis blocker received regulatory FDA approval for patients with melanoma disease progression following ipilimumab treatment and, if a BRAF V600 mutation is present, with a BRAF inhibitor.

In the first Phase I study, the KEYNOTE-001 trial, 135 patients with measurable metastatic or locally advanced unresectable melanoma were treated with pembrolizumab at a dosage of $10 \mathrm{mg} / \mathrm{kg}$ every 2 weeks, $10 \mathrm{mg} / \mathrm{kg}$ every 3 weeks, or $2 \mathrm{mg} / \mathrm{kg}$ every 3 weeks. ${ }^{45}$ Exclusion criteria were adopted regarding to melanoma of ocular origin, before treatment with PD-1/PD-L1 blocking agents, current immunosuppressive therapy, infection or autoimmune disease. Of all patients, $69 \%$ had progressed following treatment with ipilimumab, other immunotherapy, BRAF inhibitor, or chemotherapy; $31 \%$ had not received before systemic therapy. Response Evaluation Criteria in Solid Tumors (RECIST), version 1.1, revealed a response rate of $38 \%$ across all dosing cohorts, a result that did not differ in patients before treatment with ipilimumab (38\%) compared with those who had not (37\%). The highest response rate $(52 \%)$ was noted in patients who had been treated with $10 \mathrm{mg} / \mathrm{kg}$ every 2 weeks. Median PFS was $>7$ months, with $81 \%$ of patients alive at 1 year after starting treatment. Overall, the drug was well tolerated, with some drug-related adverse events ( $79 \%$ of patients), of which $66 \%$ were grade $1-2$.

Subsequently, Robert et al published the results of an open-label, international, multicenter expansion cohort of KEYNOTE-001 study ${ }^{47}$ in which eligible patients (progression of disease despite treatment with ipilimumab or a targeted kinase inhibitor) were randomized to pembrolizumab 2 or $10 \mathrm{mg} / \mathrm{kg}$ intravenously every 3 weeks until progression or intolerable toxicity. Patients with severe immune-related disease, those requiring immunosuppression, and those with a history of severe immune-related adverse effects to ipilimumab were excluded from randomization. The primary outcome, overall response, was evaluated by both RECIST and the investigator-approved immune-related response criteria. Secondary endpoints were evaluation of response duration, PFS, and OS. Finally, adverse events were broadly assessed to provide relevant side effect information that could be attributed to immune-based therapy. There was no significant difference in the safety profile, and the drug was well tolerated overall, with drug-related adverse events in $82 \%$ of each cohort patients, although only $12 \%$ presented grade 3 or 4 drug-related adverse events. By independent central review using RECIST, median PFS in the $2 \mathrm{mg} / \mathrm{kg}$ group was 22 and 14 weeks in the $10 \mathrm{mg} / \mathrm{kg}$ group. However, when assessed by immune-related response criteria, median PFS increased to 31 weeks in the $2 \mathrm{mg} / \mathrm{kg}$ treatment group and 35 weeks in the $10 \mathrm{mg} / \mathrm{kg}$ treatment group, suggesting that the conventional use of RECIST for assessing immune-based therapeutic response may underestimate actual therapeutic benefits. Kaplan-Meier estimated OS at 1 year was $58 \%$ in the $2 \mathrm{mg} / \mathrm{kg}$ treatment group compared with $63 \%$ with higher dose assigned group.

In the KEYNOTE-002 trial, 540 patients with ipilimumabrefractory advanced melanoma were randomly assigned to pembrolizumab ( $2 \mathrm{mg} / \mathrm{kg}$ every 3 weeks and $10 \mathrm{mg} / \mathrm{kg}$ every 3 weeks) or chemotherapy (carboplatin plus paclitaxel, paclitaxel alone, dacarbazine, or temozolomide per institutional standard) ${ }^{49}$ PFS assessed was significantly improved with both pembrolizumab treatment regimens compared with chemotherapy. The 6-month PFS were 34\%, 38\%, and 16\% for pembrolizumab $2 \mathrm{mg} / \mathrm{kg}, 10 \mathrm{mg} / \mathrm{kg}$, and chemotherapy, respectively. The objective response rates were $21 \%, 26 \%$, and $4 \%$, respectively, for pembrolizumab $2 \mathrm{mg} / \mathrm{kg}, 10 \mathrm{mg} / \mathrm{kg}$, and chemotherapy. Treatment was relatively well tolerated, with grade 3-5 adverse events reported in $11 \%$ and $14 \%$ of the pembrolizumab treatment arms, and $26 \%$ of those managed with chemotherapy.

Recently, Tumeh et al reported sustained tumor regression in $38 \%$ of patients in a multi-institutional, international Phase I expansion study, evaluating the safety and the clinical activity of pembrolizumab in 46 patients with advanced melanoma.$^{50}$ In this study, it was shown that preexisting $\mathrm{CD} 8^{+} \mathrm{T}$-cells, distinctly located at the invasive tumor margin, are associated with expression of the PD-1/PD-L1 immune inhibitory axis and may predict response to therapy. Specifically, in serially sampled tumors, patients responding to treatment showed proliferation of intratumoral $\mathrm{CD}^{+} \mathrm{T}$-cells that, directly, correlated with radiographic reduction in tumor size. Using a multivariate analysis, Tumeh et al established a predictive model based on CD8 expression at the invasive 
margin, indicating that tumor regression, after therapeutic PD-1 blockade, requires preexisting $\mathrm{CD}^{+} \mathrm{T}$-cells that are negatively regulated by $\mathrm{PD}-1 / \mathrm{PD}-\mathrm{L} 1-$ mediated adaptive immune resistance. ${ }^{50}$

A more recent randomized, pivotal Phase III study, KEYNOTE-006, investigating pembrolizumab compared to ipilimumab, in the first-line treatment of patients with unresectable stage III or IV advanced melanoma, was stopped early, because it met its two primary endpoints of PFS and OS. In KEYNOTE-006, pembrolizumab demonstrated a statistically significant and clinically meaningful improvement in OS and PFS compared with ipilimumab. The study randomized 834 patients to receive pembrolizumab $10 \mathrm{mg} / \mathrm{kg}$ every 3 weeks, pembrolizumab $10 \mathrm{mg} / \mathrm{kg}$ every 2 weeks, or four cycles of ipilimumab $3 \mathrm{mg} / \mathrm{kg}$ every 3 weeks. The coprimary endpoints were PFS and OS; secondary endpoints were overall response rate, duration of response, and safety, with an exploratory analysis for health-related quality of life. Tumor response was assessed at week 12, then every 6 weeks thereafter per RECIST 1.1 by independent, central, blinded radiographic review and investigator-assessed, immune-related response criteria. The safety profile of pembrolizumab in this trial was similar to the safety profile previously reported in advanced melanoma.

\section{Clinical trials - safety and tolerability}

Although pembrolizumab has been studied at three different dosing schedules detailed above, it has been approved for intravenous injection at $2 \mathrm{mg} / \mathrm{kg}$ every 3 weeks, since there did not appear to be significant differences between the dosages and schedules evaluated. Half-life of elimination for this agent is 26 days, and to date, no clinically relevant differences have been noted in its clearance in patients with renal or mild hepatic impairment.

As mentioned above, the drug is generally well tolerated with the vast majority of patients reporting reversible grade 1 or 2 drug-related adverse events, corroborating previously published data that showed a good tolerability and safety for those patients treated with anti-CTLA-4 therapy, such as ipilimumab, with a safety profile similar to that reported for ipilimumab-naïve patients. ${ }^{45,51}$ Diarrhea, endocrine adverse events, skin toxicity, myalgia, arthralgia, pyrexia, and fatigue were reported their treatment should include symptom management for mild events and the use of steroids in case of severe events. ${ }^{45,51,52}$

Adverse events of particular interest reported in literature data were of an inflammatory or autoimmune nature, including hypothyroidism, hepatic transaminitis, and pneumonitis. ${ }^{45}$ However, although uncommon, these severe adverse events of potential immune cause were successfully managed with treatment interruption or immunosuppressive therapy or both. In addition, the overall safety profile was similar in the $2 \mathrm{mg} / \mathrm{kg}$ of $10 \mathrm{mg} / \mathrm{kg}$ groups, and no deaths due to drug-related adverse events were reported. ${ }^{45,47}$ As a result, according to literature data, pembrolizumab, at both doses every 3 weeks, represents an effective treatment option not only for those patients with ipilimumab-refractory advanced melanoma but also for the vast majority of advanced melanoma patients. ${ }^{45,51}$

Although pembrolizumab is a well-tolerated drug overall, as with other immunotherapies, it is essential to maintain a high index of suspicious for immune-mediated toxicities, including pneumonitis, colitis, hepatitis, endocrynopathy ranging from hypophysitis to hyper- and hypothyroidism, and nephritis, although these are relatively infrequent.

\section{Perspectives}

As shown by other immunotherapies, the clinical benefit of pembrolizumab is long lasting. . $^{7,45,47,52}$ Obviously, this promising clinical efficacy reported thus far in several studies paves the way to its potential combination with other treatment strategies to improve the rate of responses. Indeed, the response rate seems higher than anti-CTLA-4 treatment, but there is a large room to further improvement.

The combination of BRAF inhibitors and immunotherapy may be a strategy to provide durable responses in metastatic melanoma patients. BRAF inhibitors, in fact, do not seem to impair the immune system but, on the contrary, may enhance immune activation. ${ }^{53-60}$ Several studies suggest that mutant forms of BRAF may be recognized by host immunity and could be involved in antitumor immune response. Ex vivo stimulation of lymphocytes derived from patients with melanoma, with a synthetic BRAF peptide carrying the V600E mutation led to the generation of MHC class II-restricted $\mathrm{CD}^{+}{ }^{+} \mathrm{T}$-cells specific for this peptide; this cells recognized tumor cells expressing mutant BRAF. ${ }^{61}$ Although BRAF inhibitor drugs are not designed to directly activate antitumor immune responses, there is increasing evidence to indicate enhanced antitumor immunity with use of these agents and correlation with clinical responses. Wilmott et al demonstrate increased $\mathrm{CD}^{+}$and $\mathrm{CD}^{+} \mathrm{T}$-cell infiltration in melanoma patient biopsies from patients in the early stages of treatment with Vemurafenib and Dabrafenib. ${ }^{58}$ Immune cell infiltrate rates in biopsies from patients who relapsed following treatment resembled those observed in pretreatment samples and 
correlated with the appearance of resistance against BRAF inhibitor treatment.

On a different note, pembrolizumab can be combined with T-cell therapy, which is a more complex treatment strategy involving extraction of T-cells infiltrating individual patient's tumor metastases and then re-infusion after in vitro activation and several rounds of expansion. ${ }^{62}$ Currently, a few trials are evaluating combination of anti CTLA-4 immunotherapy and T-cell therapy (NCT01701674 and NCT01988077).

Another approach, which has already been tested with another anti-PD-1 antibody (named Nivolumab, from BMS), is to combine anti-PD-1 and anti-CTLA-4 treatment. Dramatic and profound responses, which in most cases were very durable, have been observed with concurrent combination of both therapies, followed by maintenance with anti-PD-1 antibodies only. ${ }^{63}$ An astonishing $82 \%$ 1-year and 75\% 2-year survival rate has been achieved so far in a Phase Ib study. ${ }^{64}$ Combination therapy currently targeting PD-1 and CTLA-4 immune checkpoints leads to remarkable antitumor effects. Although both PD-1 and CTLA-4 dampen the T-cell activation, the in vivo effects of these drugs in humans remain to be clearly defined. In their study, Das et al evaluated combination therapy with anti-CTLA-4 and anti-PD-1 on immunologic changes in vivo. According to their data, molecules against PD-1, CTLA-4 alone, or in combination have distinct immunologic effects in vivo. ${ }^{65}$ In particular, PD-1 blockade leads to the expression of several NK-associated genes out-cells; instead, genomic signatures of CTLA-4 blockade includes the induction of several cell cycle-associated genes. ${ }^{65}$ A randomized Phase III trial of concurrent anti-PD-1/ CTLA-4 followed by PD-1 treatment only is currently ongoing (BMS Checkmate 067), but interim results have not been yet disclosed. Other trials testing sequential combinations are currently ongoing.

\section{Conclusion}

In a treatment landscape that has previously proven to be challenging for patients with advanced melanoma with progression on immune therapy, pembrolizumab offers a major paradigm shift. This monoclonal antibody is the first anti-PD-1 therapy to demonstrate a survival advantage compared to the standard of care for the first-line treatment of advanced melanoma. Results from previous studies concerning advanced melanoma showed promising activity and tolerability. Pembrolizumab is able to achieve a dual blockade (PD-L1 and PD-L2) without significant cytotoxic activity. Its promising clinical efficacy gives rise to several compelling questions in terms of potential combination with agents that are already approved, but prospective trials are needed to assess efficacy of pembrolizumab in combination with other treatments for unresectable locally advanced and metastatic melanoma.

\section{Disclosure}

The authors report no conflicts of interest in this work.

\section{References}

1. Ferlay J, Shin HR, Bray F, Forman D, Mathers C, Parkin DM. Estimates of worldwide burden of cancer in 2008: GLOBOCAN 2008. Int J Cancer. 2010;127(12):2893-2917.

2. Azoury SC, Lange JR. Epidemiology, risk factors, prevention, and early detection of melanoma. Surg Clin North Am. 2014;94(5): 945-962.

3. Balch CM, Gershenwald JE, Soong SJ, et al. Final version of 2009 AJCC melanoma staging and classification. J Clin Oncol. 2009;27(36): 6199-6206.

4. McArthur GA, Chapman PB, Robert C, et al. Safety and efficacy of vemurafenib in BRAF(V600E) and BRAF(V600K) mutation-positive melanoma (BRIM-3): extended follow-up of a phase 3, randomised, open-label study. Lancet Oncol. 2014;15(3):323-332.

5. Hauschild A, Grob JJ, Demidov LV, et al. Dabrafenib in BRAF-mutated metastatic melanoma: a multicentre, open-label, phase 3 randomised controlled trial. Lancet. 2012;380(9839):358-365.

6. Flaherty KT, Infante JR, Daud A, et al. Combined BRAF and MEK inhibition in melanoma with BRAF V600 mutations. $N$ Engl J Med. 2012;367(18):1694-1703.

7. Hodi FS, O'Day SJ, McDermott DF, et al. Improved survival with ipilimumab in patients with metastatic melanoma. N Engl J Med. 2010; 363(8):711-723.

8. Robert C, Thomas L, Bondarenko I, et al. Ipilimumab plus dacarbazine for previously untreated metastatic melanoma. $N$ Engl J Med. 2011; 364(26):2517-2526.

9. Davies H, Bignell GR, Cox C, et al. Mutations of the BRAF gene in human cancer. Nature. 2002;417(6892):949-954.

10. Zhang W. BRAF inhibitors: the current and the future. Curr Opin Pharmacol. 2015;23:68-73.

11. Robert C, Karaszewska B, Schachter J, et al. Improved overall survival in melanoma with combined dabrafenib and trametinib. N Engl J Med. 2015;372(1):30-39.

12. Chapman $P B$, Hauschild $A$, Robert $C$, et al. Improved survival with vemurafenib in melanoma with BRAF V600E mutation. NEngl J Med. 2011;364(26):2507-2516.

13. Sosman JA, Kim KB, Schuchter L, et al. Survival in BRAF V600mutant advanced melanoma treated with vemurafenib. $N$ Engl J Med. 2012;366(8):707-714.

14. Ascierto PA, Simeone E, Giannarelli D, Grimaldi AM, Romano A, Mozzillo N. Sequencing of BRAF inhibitors and ipilimumab in patients with metastatic melanoma: a possible algorithm for clinical use. J Transl Med. 2012;10:107.

15. Spagnolo F, Ghiorzo P, Queirolo P. Overcoming resistance to BRAF inhibition in BRAF-mutated metastatic melanoma. Oncotarget. 2014;5(21):10206-10221.

16. McDermott D, Haanen J, Chen TT, Lorigan P, O’Day S; MDX010-20 Investigators. Efficacy and safety of ipilimumab in metastatic melanoma patients surviving more than 2 years following treatment in a phase III trial (MDX010-20). Ann Oncol. 2013;24(10):2694-2698.

17. Robert C, Schadendorf D, Messina M, Hodi FS, O’Day S; MDX010-20 investigators. Efficacy and safety of retreatment with ipilimumab in patients with pretreated advanced melanoma who progressed after initially achieving disease control. Clin Cancer Res. 2013;19(8):2232-2239. 
18. Hanahan D, Weinberg RA. Hallmarks of cancer: the next generation. Cell. 2011;144(5):646-674.

19. Ribas A. Tumor immunotherapy directed at PD-1. $N$ Engl J Med. 2012;366(26):2517-2529.

20. Chen DS, Mellman I. Oncology meets immunology: the cancerimmunity cycle. Immunity. 2013;39(1):1-10.

21. Raval RR, Sharabi AB, Walker AJ, Drake CG, Sharma P. Tumor immunology and cancer immunotherapy: summary of the 2013 SITC primer. J Immunother Cancer. 2014;2:14.

22. Pardoll DM. The blockade of immune checkpoints in cancer immunotherapy. Nat Rev Cancer. 2012;12(4):252-264.

23. Schwartz RH. Costimulation of T lymphocytes: the role of CD28, CTLA-4, and B7/BB1 in interleukin-2 production and immunotherapy. Cell. 1992;71(7):1065-1068.

24. Egen JG, Allison JP. Cytotoxic T lymphocyte antigen-4 accumulation in the immunological synapse is regulated by TCR signal strength. Immunity. 2002;16(1):23-35.

25. Parry RV, Chemnitz JM, Frauwirth KA, et al. CTLA-4 and PD-1 receptors inhibit T-cell activation by distinct mechanisms. Mol Cell Biol. 2005;25(21):9543-9553.

26. Ishida Y, Agata Y, Shibahara K, Honjo T. Induced expression of PD-1, a novel member of the immunoglobulin gene superfamily, upon programmed cell death. EMBO J. 1992;11(11):3887-3895.

27. Keir ME, Butte MJ, Freeman GJ, Sharpe AH. PD-1 and its ligands in tolerance and immunity. Апnи Rev Immunol. 2008;26:677-704.

28. Francisco LM, Salinas VH, Brown KE, et al. PD-L1 regulates the development, maintenance, and function of induced regulatory $\mathrm{T}$ cells. J Exp Med. 2009;206(13):3015-3029.

29. Gros A, Robbins PF, Yao X, et al. PD-1 identifies the patient-specific $\mathrm{CD}^{+}$tumor-reactive repertoire infiltrating human tumors. J Clin Invest. 2014;124(5):2246-2259.

30. Dong H, Strome SE, Salomao DR, et al. Tumor-associated B7-H1 promotes T-cell apoptosis: a potential mechanism of immune evasion. Nat Med. 2002;8(8):793-800.

31. Rosenwald A, Wright G, Leroy K, et al. Molecular diagnosis of primary mediastinal B cell lymphoma identifies a clinically favorable subgroup of diffuse large B cell lymphoma related to Hodgkin lymphoma. $J$ Exp Med. 2003;198(6):851-862.

32. Gajewski TF, Louahed J, Brichard VG. Gene signature in melanoma associated with clinical activity: a potential clue to unlock cancer immunotherapy. Cancer J. 2010;16(4):399-403.

33. Thompson RH, Dong H, Lohse CM, et al. PD-1 is expressed by tumorinfiltrating immune cells and is associated with poor outcome for patients with renal cell carcinoma. Clin Cancer Res. 2007;13(6):1757-1761.

34. Zhang Y, Huang S, Gong D, Qin Y, Shen Q. Programmed death-1 upregulation is correlated with dysfunction of tumor-infiltrating $\mathrm{CD} 8^{+}$ T lymphocytes in human non-small cell lung cancer. Cell Mol Immunol. 2010;7(5):389-395.

35. Ahmadzadeh M, Johnson LA, Heemskerk B, et al. Tumor antigenspecific CD8 T cells infiltrating the tumor express high levels of PD-1 and are functionally impaired. Blood. 2009;114(8):1537-1544.

36. Shi F, Shi M, Zeng Z, et al. PD-1 and PD-L1 upregulation promotes $\mathrm{CD} 8(+) \mathrm{T}$-cell apoptosis and postoperative recurrence in hepatocellular carcinoma patients. Int J Cancer. 2011;128(4):887-896.

37. Zou W, Chen L. Inhibitory B7-family molecules in the tumour microenvironment. Nat Rev Immunol. 2008;8(6):467-477.

38. Taube JM, Anders RA, Young GD, et al. Colocalization of inflammatory response with B7-h1 expression in human melanocytic lesions supports an adaptive resistance mechanism of immune escape. Sci Transl Med. 2012;4(127):127ra37.

39. Atefi M, Avramis E, Lassen A, et al. Effects of MAPK and PI3K pathways on PD-L1 expression in melanoma. Clin Cancer Res. 2014;20(13): 3446-3457.

40. Taube JM, Klein A, Brahmer JR, et al. Association of PD-1, PD-1 ligands, and other features of the tumor immune microenvironment with response to anti-PD-1 therapy. Clin Cancer Res. 2014;20(19):5064-5074.
41. Barber DL, Wherry EJ, Masopust D, et al. Restoring function in exhausted CD8 T cells during chronic viral infection. Nature. 2006;439(7077): 682-687.

42. Iwai Y, Ishida M, Tanaka Y, Okazaki T, Honjo T, Minato N. Involvement of PD-L1 on tumor cells in the escape from host immune system and tumor immunotherapy by PD-L1 blockade. Proc Natl Acad Sci U S A. 2002;99(19):12293-12297.

43. Nakanishi J, Wada Y, Matsumoto K, Azuma M, Kikuchi K, Ueda S. Overexpression of B7-H1 (PD-L1) significantly associates with tumor grade and postoperative prognosis in human urothelial cancers. Cancer Immunol Immunother. 2007;56(8):1173-1182.

44. Thompson RH, Webster WS, Cheville JC, et al. B7-H1 glycoprotein blockade: a novel strategy to enhance immunotherapy in patients with renal cell carcinoma. Urology. 2005;66(5 Suppl):10-14.

45. Hamid O, Robert C, Daud A, Hodi FS, et al. Safety and tumor responses with lambrolizumab (anti-PD-1) in melanoma. N Engl J Med. 2013;369(2):134-144.

46. Tolcher AW, Messersmith WA, Mikulski SM, et al. Phase I study of RO4929097, a gamma secretase inhibitor of Notch signaling, in patients with refractory metastatic or locally advanced solid tumors. J Clin Oncol. 2012;30(19):2348-2353.

47. Robert C, Ribas A, Wolchok JD, et al. Anti-programmed-deathreceptor-1 treatment with pembrolizumab in ipilimumab-refractory advanced melanoma: a randomised dose-comparison cohort of a phase 1 trial. Lancet. 2014;384(9948):1109-1117.

48. Ribas A, Hodi FS, Kefford R, et al. Efficacy and safety of the anti-PD-1 monoclonal antibody pembrolizumab (MK-3475) in 411 patients (pts) with melanoma (MEL) (Abstract LBA9000). In: American Society of Clinical Oncology (ASCO) Meeting; 2014.

49. Ribas A, Puzanov I, Dummer R, et al. A Randomized Controlled Comparison of Pembrolizumab and Chemotherapy in Patients With Ipilimumab-Refractory Melanoma. Presented at: Society for Melanoma; 2014.

50. Tumeh PC, Harview CL, Yearley JH, et al. PD-1 blockade induces responses by inhibiting adaptive immune resistance. Nature. 2014; 515(7528):568-571.

51. Topalian SL, Hodi FS, Brahmer JR, et al. Safety, activity, and immune correlates of anti-PD-1 antibody in cancer. $N$ Engl J Med. 2012; 366(26):2443-2454.

52. Topalian SL, Sznol M, McDermott DF, et al. Survival, durable tumor remission, and long-term safety in patients with advanced melanoma receiving nivolumab. J Clin Oncol. 2014;32(10):1020-1030.

53. Hong DS, Vence L, Falchook G, et al. BRAF(V600) inhibitor GSK2118436 targeted inhibition of mutant BRAF in cancer patients does not impair overall immune competency. Clin Cancer Res. 2012;18(8): 2326-2335.

54. Comin-Anduix B, Chodon T, Sazegar H, et al. The oncogenic BRAF kinase inhibitor PLX4032/RG7204 does not affect the viability or function of human lymphocytes across a wide range of concentrations. Clin Cancer Res. 2010;16(24):6040-6048.

55. Frederick DT, Piris A, Cogdill AP, et al. BRAF inhibition is associated with enhanced melanoma antigen expression and a more favorable tumor microenvironment in patients with metastatic melanoma. Clin Cancer Res. 2013;19(5):1225-1231.

56. Boni A, Cogdill AP, Dang P, et al. Selective BRAFV600E inhibition enhances T-cell recognition of melanoma without affecting lymphocyte function. Cancer Res. 2010;70(13):5213-5219.

57. Liu $\mathrm{C}$, Peng $\mathrm{W}, \mathrm{Xu} \mathrm{C}$, et al. BRAF inhibition increases tumor infiltration by $\mathrm{T}$ cells and enhances the antitumor activity of adoptive immunotherapy in mice. Clin Cancer Res. 2013;19(2):393-403.

58. Wilmott JS, Long GV, Howle JR, et al. Selective BRAF inhibitors induce marked T-cell infiltration into human metastatic melanoma. Clin Cancer Res. 2012;18(5):1386-1394.

59. Cooper ZA, Juneja VR, Sage PT, et al. Response to BRAF inhibition in melanoma is enhanced when combined with immune checkpoint blockade. Cancer Immunol Res. 2014;2(7):643-654. 
60. Callahan MK, Masters G, Pratilas CA, et al. Paradoxical activation of T cells via augmented ERK signaling mediated by a RAF inhibitor. Cancer Immunol Res. 2014;2(1):70-79.

61. Sharkey MS, Lizée G, Gonzales MI, Patel S, Topalian SL. CD4(+) $\mathrm{T}$-cell recognition of mutated B-RAF in melanoma patients harboring the V599E mutation. Cancer Res. 2004;64(5):1595-1599.

62. Donia M, Eva Ellebæk, Inge Marie Svane. Tumor Immunology and Immunotherapy. In: Robert C Rees, editor. Adoptive T-cell therapy using TILs for the treatment of metastatic melanoma. New York: Oxford University Press; 2012:327-330.
63. Wolchok JD, Kluger H, Callahan MK, et al. Nivolumab plus ipilimumab in advanced melanoma. $N$ Engl J Med. 2013;369(2):122-133.

64. Sznol M, Kluger HM, Callahan MK, et al. Survival, response duration, and activity by BRAF mutation (MT) status of nivolumab (NIVO, anti-PD-1, BMS-936558, ONO-4538) and ipilimumab (IPI) concurrent therapy in advanced melanoma (MEL). J Clin Oncol. 2014;32(15 suppl): abstr LBA9003.

65. Das R, Verma R, Sznol M, et al. Combination therapy with anti-CTLA-4 and anti-PD-1 leads to distinct immunologic changes in vivo. J Immunol. 2015;194(3):950-959.

\section{Publish your work in this journal}

OncoTargets and Therapy is an international, peer-reviewed, open access journal focusing on the pathological basis of all cancers, potential targets for therapy and treatment protocols employed to improve the management of cancer patients. The journal also focuses on the impact of management programs and new therapeutic agents and protocols on

\section{Dovepress}

patient perspectives such as quality of life, adherence and satisfaction. The manuscript management system is completely online and includes a very quick and fair peer-review system, which is all easy to use. Visit http://www.dovepress.com/testimonials.php to read real quotes from published authors.

\footnotetext{
Submit your manuscript here: http://www.dovepress.com/oncotargets-and-therapy-journal
} 